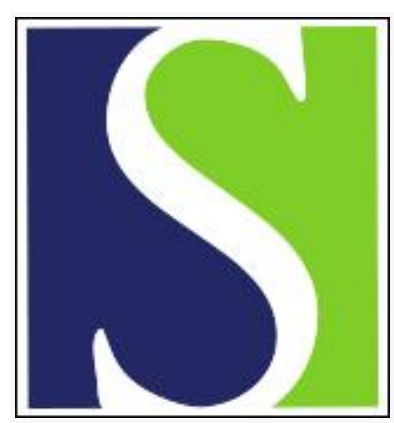

Scand J Work Environ Health 1986;12(4):428-431

https://doi.org/10.5271/sjweh.2118

Issue date: Aug 1986

Assessing the severity of the neurological component of the hand-arm vibration syndrome.

by Brammer AJ, Taylor W, Piercy JE

This article in PubMed: www.ncbi.nlm.nih.gov/pubmed/3775334

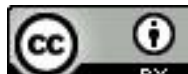




\title{
Assessing the severity of the neurological component of the hand-arm vibration syndrome
}

\author{
by Anthony J Brammer, PhD, ${ }^{1}$ William Taylor, MD, DSc, ${ }^{2}$ Joseph E Piercy, PhD ${ }^{1}$
}

\begin{abstract}
BRAMMER AJ, TAYLOR W, PIERCY JE. Assessing the severity of the neurological component of the hand-arm vibration syndrome. Scand J Work Environ Health 12 (1986) 428-431. In view of the observation in epidemiologic studies that the neurological component of the hand-arm vibration syndrome develops first, a conservative measure of the presence and progression of the syndrome may be obtained from the degree of numbness and loss of fine touch. Degraded tactile spatial resolution, as measured by step and gap detection with an esthesiometer, has been found, on the average, in stages 2 and 3 of the Taylor-Pelmear classification for vibration-induced white finger (VWF). Present evidence suggests that this occurrence is due to the degeneration of slowly adapting (type I) and, possibly, fast adapting (type I) mechanoreceptors, and/or their nerve fibers. The apparatus commonly employed for measuring vibrotactile perception thresholds in clinical studies records the sensitivity of fast adapting type II mechanoreceptors (pacinian corpuscles), which play no role in step or gap detection. They may, however, provide early evidence of neurological changes occurring in the syndrome, as a significant degradation in the threshold of fast adapting type II receptors appears to develop, on the average, before stage 2 of VWF.
\end{abstract}

Key terms: edge detection, esthesiometer, fine touch, vibrotactile perception, white fingers.

Repeated exposure of the hand to vibration may result in a complex of neurological, vascular, and musculoskeletal disturbances in the hand-arm system. The most common of these are classified by severity in the Taylor-Pelmear stages of vibration-induced white finger (VWF), which focus on neurological symptoms and vascular signs (8). Although the earliest clinical manifestations - tingling, numbness, and fingertip blanching (stages $\mathrm{O}_{\mathrm{T}}, \mathrm{O}_{\mathrm{N}}$ and 1 , respectively) - are episodic in nature and usually judged to be of little consequence by those affected, prolonged exposure to vibration may lead to sufficient impairment of tactile function to restrict the ability to perform manual work.

A serious obstacle to quantifying the severity of these disorders has been the difficulty of establishing clinical tests for aiding diagnosis, an almost complete reliance being placed at present on the worker's occupational history and (subjective) recollection of signs and symptoms. Thus in contrast to industrial noise exposure, for which audiometric techniques provide a measure of hearing threshold degradation, there is no corresponding objective test for any component of the handarm vibration syndrome. In view of the observation in epidemiologic studies that the neurological symptoms develop first (1), a conservative measure of the presence and progression of the syndrome may be obtained from the degree of numbness and loss of fine

1 Division of Physics, National Research Council of Canada, Ottawa, Canada.

2 Department of Community Medicine, University of Dundee, Dundee, Scotland.

Reprint requests to: Dr AJ Brammer, Division of Physics, National Research Council of Canada, Montreal Road, Ottawa, Ontario K1A OR6, Canada. touch. Hence methods for quantifying tactile sensory dysfunction within the fingers become prime candidates for objective tests.

In this paper, the degradation of fine touch in vibration-exposed workers and its underlying mechanisms are explored. The clinical findings employed in this analysis are based solely on medical evaluations and the corresponding classification of symptoms performed by one of us (WT), to insure that the TaylorPelmear staging, which inevitably serves as the measure of severity, has been consistently applied. Only a summary of the sensory measurements conducted by ourselves and others are included; details of the experimental procedures and results are to be found elsewhere $(2,7,9)$.

\section{Summary of results}

\section{Vibrotactile perception thresholds}

An attempt to correlate vibrotactile perception thresholds with the Taylor-Pelmear classification of VWF has been made for a population of forest workers consisting of 196 persons exposed to chain-saw vibration, and 72 referents (controls) (9). The apparatus consisted of a small vibration exciter and accelerometer mounted on a beam balance. This arrangement provided a constant contact force of $0.15 \mathrm{~N}$ between a cylindrical, flat-topped probe, $22 \mathrm{~mm}$ in diameter, and a fingertip. The hand and arm rested horizontally on a plate containing a hole through which the vertically mounted probe contacted the skin.

The results are plotted as displacement amplitude (zero-peak) in decibels re $1 \mu \mathrm{m}$ in figures 1 and 2. Mean 


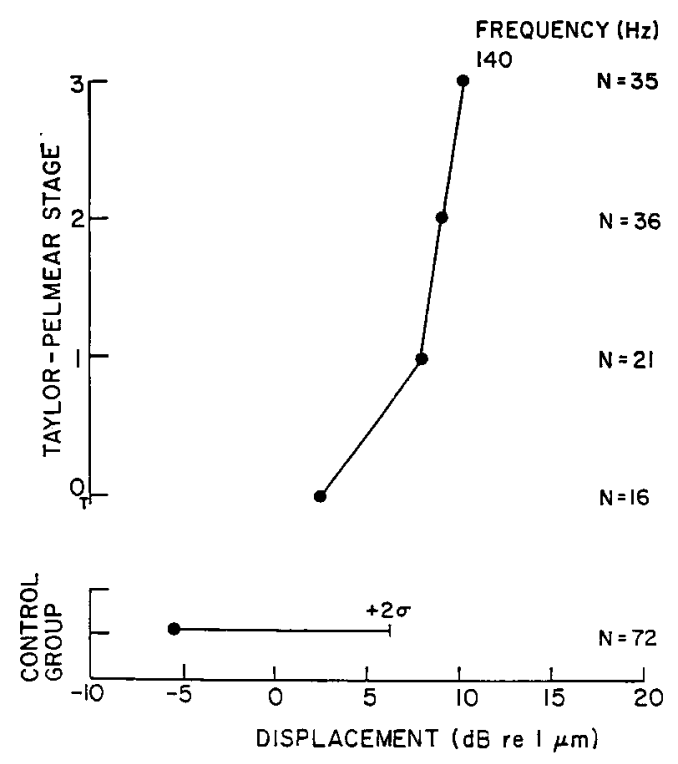

Figure 1. Vibrotactile perception thresholds at $140 \mathrm{~Hz}$ for the referents (control group) (mean and mean plus two standard deviations) and symptomatic chain-saw operators, grouped by their Taylor-Pelmear stage of vibration-induced white finger (mean).

thresholds at one frequency $(140 \mathrm{~Hz})$ are shown for the reference group and for all symptomatic workers by stage of VWF in figure 1 and for all frequencies at which measurements were performed $(80,140,250$ and $400 \mathrm{~Hz}$ ) in figure 2 . For the reference group, the difference in perception threshold between individuals is indicated by the horizontal line and bar in figure 1 and by the vertical lines and bars in figure 2 . Corresponding standard deviations for the vibrationexposed workers are not available by stage of VWF, but they were similar in magnitude to those shown in figures 1 and 2 when the thresholds for all chain-saw operators were combined. These diagrams reveal that the mean thresholds for workers experiencing vasospasms (stages 1-3) exceed those of the referents by more than two standard deviations $(2 \sigma)$.

\section{Tactile spatial resolution}

In our most recent attempt to correlate tactile perception with the severity of VWF, two elements of spatial resolution were measured in a series of severe cases referred to one of us (WT) for medical evaluation (7). All were from occupations in which significant vibration exposure occurs (pneumatic drill operators, pedestal grinders, and swagers). None of these cases responded positively to clinical tests for carpal tunnel syndrome.

The esthesiometer designed by Carlson et al was employed for the sensory measurements using surfaces containing a step or groove of continuously varying height or width (d), respectively. (See the insets to figure 3) (2). These surfaces are mounted on plates

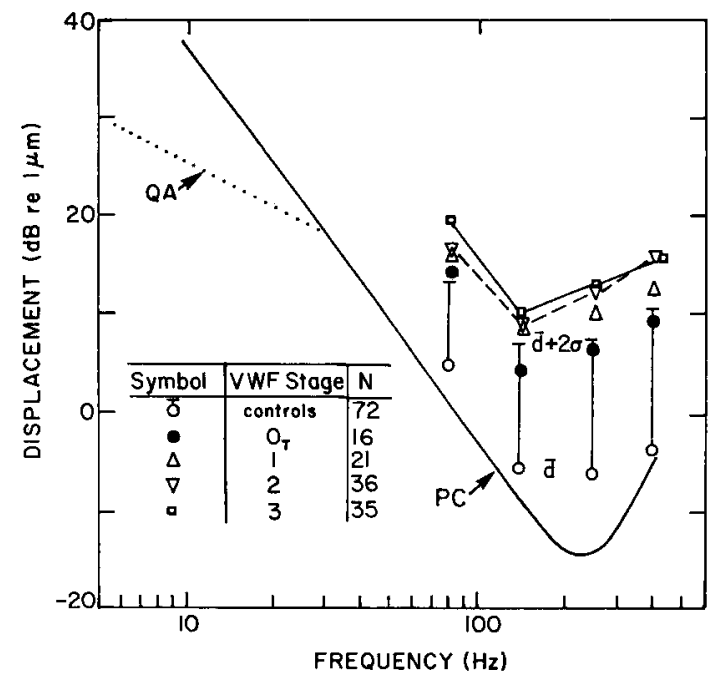

Figure 2. Vibrotactile perception thresholds of the referents (controls) (mean and mean plus two standard deviations) and symptomatic chain-saw operators, grouped by their TaylorPelmear stage of vibration-induced white finger (VWF) (mean) The continuous and dotted lines show the thresholds of the fast adapting type II [or pacinian corpuscles (PC)] and fast adapting type I [or quickly adapting (QA)] mechanoreceptors, respectively, reported by Mountcastle et al (5).

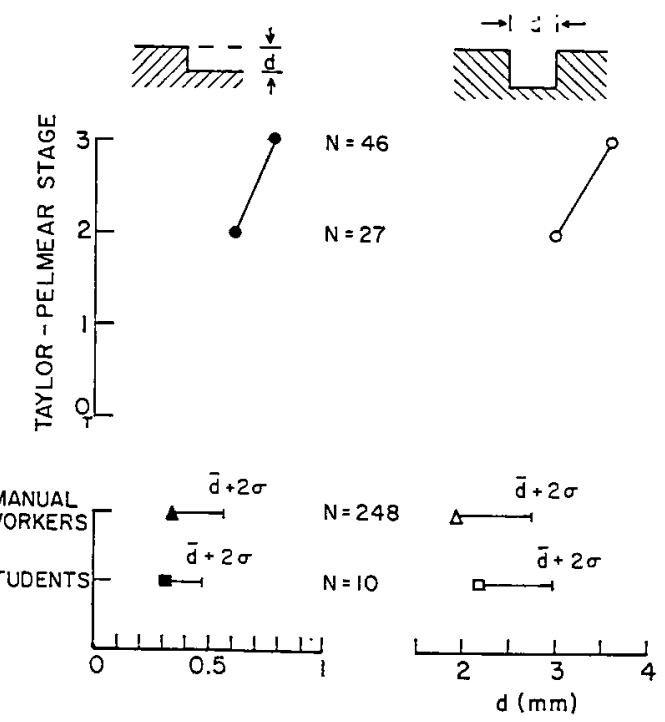

Figure 3. Detection threshold (d) of step height (shown to the left) and gap width (shown to the right) for manual workers not exposed to vibration and students (mean and mean plus two standard deviations) and 73 cases of Taylor-Pelmear stage 2 and 3 (mean).

which, in turn, are supported on sprung tracks, so that the step or groove may be drawn across a restrained fingertip with a constant contact force (IN).

The mean detection thresholds for 73 severe cases, all of whom were judged to be in stage 2 or 3 of VWF, are shown by the circles in figure 3 . They are compared 
with thresholds from 248 manual workers not exposed to vibration (the triangles) and those from 10 male students (the squares), obtained previously with the same apparatus by the National Institute for Occupational Safety and Health (Behrens, personal communication 1983, and reference 2). As before, the range of thresholds found in unexposed persons is indicated by horizontal lines and bars. They demonstrate that the mean thresholds for cases in stages 2 and 3 exceed those of unexposed persons by at least two and four standard deviations, respectively.

\section{Discussion}

\section{Interpretation of the tactile results}

There is evidence from finger biopsies and postmortem tissue sections that the neurological changes associated with chronic vibration exposure are occurring primarily in the mechanoreceptors and peripheral nerve fibers $(6,11)$. These observations are supported by laboratory experiments in which individual mechanoreceptor firings and the corresponding psychophysical perception thresholds were recorded simultaneously when the receptor was stimulated by vibration. In these measurements on the fingertips of normal persons, similar temporary increases in vibration intensity were required to elicit both neural firings and perception after the provocative vibration exposure (3).

Up to four types of mechanoreceptors may be involved in the sense of touch in glabrous skin (10). They are two types of fast adapting [FA I and FA II (pacinian corpuscles)] and two types of slowly adapting (SA I and SA II) receptors. Neurophysiological studies of individual mechanoreceptor firings in monkey fingertips reveal that SA I and FA I receptors produce a variation in the rate of neural firings in response to a ridged surface (4). This response to sharp edges, which is clearly resolved by the SA I receptors when the edges are separated by more than $1 \mathrm{~mm}$, is not found in neural firings from the FA I receptors until the separation is approximately $5 \mathrm{~mm}$. In the same experiment, the ability of the FA II receptors to resolve edges was even worse. Hence edge and gap detection, elements of tactile spatial resolution measured by an esthesiometer, appear to be determined by the performance of SA I and, possibly, FA I receptors. The data of figure 3 thus suggest that, on the average, these receptors and/or their associated nerve fibers are adversely affected in workers suffering from stage 2 or 3 of VWF.

An interpretation of the vibrotactile perception thresholds recorded from the population of vibrationexposed workers may now be obtained by reference to figure 2 . Also shown in this diagram by the continuous and dotted lines are the results of carefully controlled laboratory measurements by Mountcastle and co-workers (5), in which psychophysical vibrotactile perception thresholds were correlated with receptor type. It is evident from both the frequency range and trend of the clinical data that they represent a measure of the sensitivity of the FA II receptors rather than of the sensors believed to be responsible for two critical components of tactile spatial resolution.

Close inspection of figures 1 and 2 also reveals that most degradation of the thresholds of the FA II receptors occurs prior to the occurrence of extensive finger blanching (ie, before stage 2 ). This phenomenon takes place long before workers complain of difficulty with tasks involving fine work (stage 3 ) and may reflect early changes in the end organs or nerve fibers. The link between degraded thresholds of the FA II receptors and tactile spatial perception, however, remains unclear, though there is some evidence to suggest a role for these receptors in determining surface texture (4).

\section{Classification of signs and symptoms of vibration-induced white finger}

The Taylor-Pelmear clinical classification of signs and symptoms, which has served as the basis of this analysis, emphasizes the vascular component of VWF. As it is now believed that the various components of the hand-arm vibration syndrome may develop independently, a correlation between an objective test based on tactile sensation (a measure of neurological dysfunction) and the original Taylor-Pelmear stages cannot always be expected. Accordingly, it is appropriate to revise the classification, perhaps along the lines suggested in table 1 , to include separately neurological and vascular stages (identified by $\mathrm{N}$ and $\mathrm{V}$, respectively).

Table 1. Proposed revisions to the Taylor-Pelmear clinical stages of vibration-induced white finger.

\begin{tabular}{lll}
\hline Stage & Signs and symptoms & Interference with activities \\
\hline ON, oV & No signs or symptoms & None \\
1N & Intermittent tingling and/or numbness & None \\
$1 \mathrm{~V}$ & Episodic blanching of one or more fingertips & None \\
$2 \mathrm{~N}$ & Intermittent numbness; reduced tactile & Possible interference with activities involving \\
& perception & fine tasks \\
$2 \mathrm{~V}$ & Episodic blanching of one or more fingers, & Some interference with social activities \\
$3 \mathrm{~N}$ & usually confined to winter & \\
& Degraded tactile resolution; intermittent & Interference with activities involving fine \\
$3 \mathrm{~V}$ & numbness & tasks at work and at home \\
& Extensive finger blanching, frequent & Restriction of hobbies and social activities \\
& episodes summer and winter & to avoid vasospasms
\end{tabular}


These stages parallel those of the original classification and attempt to separate the interference with activities, as well as the signs and symptoms, into those of primarily neurological or vascular origin.

Such a classification would allow for the introduction of quantitative measures of tactile sensory deficit by means of objective tests without the need for a correlation with the severity and frequency of the vasospastic episodes. In this way, the permanent neurological changes thought to be associated with damage to the mechanoreceptors and peripheral nerve fibers may be completely dissociated from the somewhat capricious episodes of finger blanching.

\section{Conclusions}

A degradation in fine touch, as measured by the perception of step height and gap width, two elements of tactile spatial resolution, appears to occur, on the average, in workers suffering from stages 2 and 3 of VWF in the Taylor-Pelmear classification. There is evidence from pathological, neurophysiological, and psychophysical sources to suggest that this change is probably due to degeneration of SA I and possibly FA I mechanoreceptors and/or their associated nerve fibers.

The apparatus commonly employed for vibrotactile measurements in past clinical studies records the sensitivity of the FA II mechanoreceptors. These receptors play no role in the detection of step height or gap width. They may, however, provide early evidence of neurological changes occurring in the syndrome, as a significant degradation of the threshold of the FA II receptors appears to develop, on the average, before stage 2 of VWF.

\section{Acknowledgments}

The authors are indebted to the United States National Institute for Occupational Safety and Health, Physical
Agents Branch, and, in particular, to Ms V Behrens for performing additional statistical analyses of their esthesiometric data at our request.

\section{References}

1. Behrens V, Taylor W, Wasserman DE. Vibration syndrome in workers using pneumatic chipping and grinding tools. In: Brammer AJ, Taylor W, ed. Vibration effects on the hand and arm in industry. John Wiley \& Sons, New York, NY 1982, pp 147-155.

2. Carlson WS, Samueloff S, Taylor W, Wasserman DE. Instrumentation for measurement of sensory loss in the fingertips. J Occup Med 21 (1979) 260-264.

3. Johansson RS, Lundström R. Lokala vibrationer och handens taktila känsel. Institutionen för fysiologi, Umeå Universitet, Umeå 1982, pp 1-69. (ASF rapport).

4. Johnson KO, Phillips JR. Spatial and nonspatial neural mechanisms underlying tactile spatial discrimination. In: von Euler C, Franzén O, Lindblom U, Ottoson D, ed. Somatosensory mechanisms. MacMillan Press, London 1984, pp 237-248.

5. Mountcastle VB, Lamotte RH, Carli G. Detection thresholds for stimuli in humans and monkeys: Comparison with threshold events in mechanoreceptive afferent nerve fibers innervating the monkey hand. J Neurophysiol 35 (1972) 122-136.

6. Takeuchi T, Imanishi H. Histopathologic observations in finger biopsy from thirty patients with Raynaud's phenomenon of occupational origin. J Kumamoto Med Soc 58 (1984) 56-70.

7. Taylor W, Ogston SA, Brammer AJ. A clinical assessment of seventy-eight cases of the hand-arm vibration syndrome. Scand J Work Environ Health 12 (1986) $265-268$.

8. Taylor W, Pelmear PL, ed. Vibration white finger in industry. Academic Press, London 1975, p xxi.

9. Taylor W, Pelmear PL, Pearson J. Raynaud's phenomenon in forestry chain saw operators. In: Taylor W, ed. The vibration syndrome. Academic Press, London 1974, pp 122-139.

10. Vallbo AB, Johansson RS. Properties of cutaneous mechanoreceptors in the human hand related to touch sensation. Human Neurobiol 3 (1984) 3-14.

11. Walton K. The pathology of Raynaud's phenomenon of occupational origin. In: Taylor W, ed. The vibration syndrome. Academic Press, London 1974, pp 113-115. 